\title{
Repetitive Transcranial Magnetic Stimulation Alleviates Neurological Deficits After Cerebral Ischemia Through Interaction Between RACK1 and BDNF exon IV by the Phosphorylation-Dependent Factor MeCP2
}

\author{
Hongzhan $\mathrm{Li}^{1}$ • Jianqing Shang ${ }^{1} \cdot$ Chengliang Zhang ${ }^{2} \cdot{\text { Rulan } \mathrm{Lu}^{2}}^{2}$ Junpao Chen ${ }^{1} \cdot$ Xianju Zhou ${ }^{1,2}$
}

Published online: 7 January 2020

(C) The American Society for Experimental NeuroTherapeutics, Inc. 2019

\begin{abstract}
Repetitive transcranial magnetic stimulation (rTMS) is acknowledged as a form of neurostimulation, especially for functional recovery. The foundational knowledge of molecular mechanism is limited regarding its role in cerebral ischemia, for which the present study was designed. Primary neurons were treated with oxygen-glucose deprivation (OGD) and repetitive magnetic stimulation (rMS), in which brain-derived neurotrophic factor (BDNF) and transcription of BDNF exons were examined. Then, adenovirus vectors carrying siRACK1 sequence were delivered to primary neurons, followed by detection of the transcription of $B D N F$ exons and the extent of methyl $\mathrm{CpG}$ binding protein 2 (MeCP2) phosphorylation. Results showed that BDNF and the transcription of BDNF exons were upregulated by rMS and OGD treatment, but decreased by extra treatment of RACK1 siRNA. Then, the mechanism investigations demonstrated that rMS increased the extent of MeCP2 phosphorylation to promote the interaction between RACK1 and BDNF exon IV. The aforementioned findings were further confirmed in vivo in middle cerebral artery occlusion (MCAO)-induced rat models, as indicated by improved neurological functions and reduced area of cerebral infarction. The study offers potential evidence for improvement of neurological deficits, highlighting the important role of rTMS for treatment of cerebral ischemia.
\end{abstract}

Key Words Repetitive transcranial magnetic stimulation - cerebral ischemia $\cdot$ BDNF RACK1 $\cdot$ MeCP2 phosphorylation · neurological function

\section{Introduction}

Cerebral ischemia is the most prevalent cause of acute cerebral infarction across the world with high mortality rates [1]. Neurologic deficits relative to cerebral ischemia can be attributed to dysfunction of several neurotransmitter systems, including the cholinergic and glutamatergic systems [2]. In

Hongzhan Li and Jianqing Shang contributed equally to this work.

Xianju Zhou

xianju_zhou@yahoo.com

1 Department of Neurology, Integrated Hospital of Traditional Chinese Medicine, Southern Medical University, No. 13, Shiliugang Road, Guangzhou 510315, Guangdong Province, China

2 Department of Neurology, The Affiliated Changzhou No. 2 People's Hospital of Nanjing Medical University, No. 29, Xinglong Alley, Changzhou 213003, Jiangsu Province, China recent years, noninvasive brain stimulation has been widely applied for the regulation of brain functions and neuropsychiatric conditions [3]. For instance, evidence suggests that repetitive transcranial magnetic stimulation (rTMS) can be used to improve cognitive decline in patients with Alzheimer's disease [4], and the recovery of neurological functions after the occurrence of cerebral ischemia [5]. Importantly, the improvement of high-frequency rTMS on neurological functional recovery in ischemic rat models has been linked to brain-derived neurotrophic factor (BDNF) [6]. BDNF that can be produced throughout the brain is capable of regulating neuronal activity and normal neuronal function as well as plays an important role in protection and recovery after stroke [7]. Likewise, BDNF has been highlighted to serve as a contributor in rTMS-produced amelioration of hippocampus-dependent cognitive impairment in rats with ischemic stroke [8]. Therefore, it is reasonable to infer that rTMS may be applied to promote neurogenesis by the neurorehabilitation effects of BDNF. 
Additionally, BDNF stimulation has been suggested to induce the receptor for activated $\mathrm{C}$ kinase 1 (RACK1) to mediate the local translation of synaptic protein, promoting neural development [9]. RACK1, a scaffold protein consisting of 7 tryptophan-aspartate (WD) 40 domains, is capable of regulating interaction between proteins [10]. Targeting the RACK1 signaling pathway has further been highlighted to serve as a therapeutic approach for neurodevelopment disorders [11]. In addition, the tumor necrosis factor- $\alpha$ receptor/RACK1/embryonic ectoderm development signaling pathway also play a role in ischemia-associated neuronal damage [12], suggesting the possible involvement of RACK1 in BDNF-mediated cerebral ischemia. Intriguingly, a previous study revealed that rTMS to cortex promotes the function of BDNFtropomyosin-related kinase B (TrkB)- $N$-methyl-Daspartic acid (NMDA) receptor axis in both cortex and lymphocytes [13]. Besides, the neuroprotective effects of NMDA have been found to reduce accompanied by decreased BDNF protein levels in association with neuronal survival [14]. Moreover, BDNF induced methyl $\mathrm{CpG}$ binding protein $2(\mathrm{MeCP} 2)$ phosphorylation at $\mathrm{S} 86$ and S274, regulating the responses of neuronal chromatin to diverse stimuli [15]. MeCP2, known as an epigenetic factor that is highly abundant in the brain, can bind to both DNA nonmethylated and methylated regions of chromatin, and may be responsible for mediation of responses to immunity and inflammation $[16,17]$. As previously described, BDNF promoter IV can be activated by releasing the transcription repressor, $\mathrm{MeCP} 2$, from the promoter leading to DNA demethylation [16]. Hereby, we hypothesize whether rTMS could improve neurological functions in cerebral ischemia involving the interaction of BDNF, RACK1, and MeCP2. In the current study, we aim to employ a cellular ischemia model OGD in the primary rat neurons and in vivo cerebral ischemia rat model middle cerebral artery occlusion (MCAO) to investigate the role of rTMS in the treatment of cerebral ischemia.

\section{Materials and Methods}

\section{Experimental Animals}

A total of 75 adult male Sprague-Dawley (SD) rats at specific pathogen free (SPF) level (aged 8-12 weeks, weighing 200-250 g) were obtained from the Hunan SJA Laboratory Animal Co., Ltd. (license key: SCXK (xiang) 2009-0004, Changsha, Hunan, China). All rats were raised under condition of 12-h light/darkness with free access to food and water. Ten rats were selected as normal controls, and the remaining 60 rats were used to establish the cerebral ischemic model. All experimental animals were euthanized by $\mathrm{CO}_{2}$ asphyxiation.

\section{Adenovirus Vector Construction}

The RACK1 sequence was inserted into AAV5-LacZ vector to obtain adenovirus vectors for upregulating RACK1 expression. The AAV5-LacZ cosmid vectors, provided by the adenovirus expression vector kit (TaKaRa Biotechnology Inc., Japan), were cotransfected with relevant reagents into 293 cells in accordance with the manufacturer's instructions. The wildtype strain was screened from the obtained recombinant adenovirus at passage 1 by differential infection method using HeLa cells and 293 cells, followed by amplification using Dulbecco's Modified Eagle Medium (DMEM) to harvest AAV5-LacZ adenovirus at passage 4 with higher titer.

\section{Culture and Treatment of Primary Neurons}

The cortex, striatum, and hippocampus were separated from rat brains, washed with D-Hanks for 3 times, respectively, sliced into pieces and detached with $2.5 \mathrm{~g} / \mathrm{L}$ trypsin at $37^{\circ} \mathrm{C}$ for $10 \mathrm{~min}$. The detachment was eliminated by the addition of DMEM containing $10 \%$ fetal bovine serum (FBS). The elimination agent was removed through centrifugation at $179 \times \mathrm{g}$ for $10 \mathrm{~min}$, and this step was repeated again. After filtration, the filtrate was centrifuged at $179 \times g$ for $10 \mathrm{~min}$ and washed with D-Hanks for 2 times. A single cell suspension was produced by DMEM containing $10 \%$ bovine serum albumin and $10 \%$ B-27. With cell concentration adjusted to $2 \times 10^{5}$ cells/ $\mathrm{mL}$, the cells were seeded in a 96-well plate coated with $0.05 \mathrm{~g} / \mathrm{L}$ poly-L-lysine and incubated at $37{ }^{\circ} \mathrm{C}$ with $5 \%$ $\mathrm{CO}_{2}$ in air. The following day, the cortex, striatum, and hippocampus neurons were cultured in oxygen-glucose deprivation (OGD)-treated condition (sugar-free DMEM, $5 \% \mathrm{O}_{2},<1 \% \mathrm{CO}_{2}$, and $95 \% \mathrm{~N}_{2}$ ) for $1 \mathrm{~h}$ to obtain one-time OGD neurons. In order to simulate a condition of recurrent ischemia, OGD neurons were required for two times. Then, $60 \mathrm{~min}$ after the first time of OGD, the sample was cultured in normal medium for $3 \mathrm{~h}$ and treated with OGD for $60 \mathrm{~min}$, followed by normal culture. The primary cultured neurons were transfected or infected with sequences of Mock, siRACK1 (20 nt, GACCATCATCATGTGGAAGC), siNC, AV-oe-NC, and AV-oe-RACK1 [18], respectively. Next, the cells were seeded in a 6 -well plate $48 \mathrm{~h}$ prior to transfection. 
When cell confluence reached $80-90 \%$, cell transfection was performed according the instructions of lipofectamine 2000 (11668-019, Invitrogen, NY, CA, USA). Following incubation at $37{ }^{\circ} \mathrm{C}$ with $5 \% \mathrm{CO}_{2}$ in air for $6-8 \mathrm{~h}$, the culture was continued for $24-48 \mathrm{~h}$ in complete medium for subsequent experimentation.

\section{Focal Cerebral Ischemia Model Induced by Middle Cerebral Artery Occlusion in Rats}

Focal cerebral ischemia model was induced by MCAO as previously described $[19,20]$. General anesthesia was performed using 3\% pentobarbital sodium (P3761, Sigma-Aldrich Chemical Company, St Louis, MO, USA) and sustained by inhalation of $1.5 \%$ isofluothane. The rats were placed in an incubator after surgery with their body temperature maintained at $36.5-37{ }^{\circ} \mathrm{C}$. A total of 63 rats were used for modeling, and 3 rats died after the completion of surgery. The remaining 60 rats were subjected to different treatments with 12 rats for each, including MCAO, MCAO $+1 \mathrm{~Hz}$ rTMS, MCAO $+10 \mathrm{~Hz}$ rTMS, NVP-AAM007+MCAO + $1 \mathrm{~Hz}$ rTMS, and Ro25-6981 + MCAO +1 Hz rTMS. An additional 12 rats were regarded as normal controls without any treatment (mock group).

\section{rTMS Protocols and Neurological Evaluation}

The rTMS instrument (Yiruide Medical Instrument Co., Ltd. Wuhan, Hubei, China), was introduced for intervention with the highest frequency of $100 \mathrm{~Hz}$, and external diameter of circular stimulation coil being $6 \mathrm{~cm}$. The primary cultured neurons were placed under rTMS and treated with stimulation of low and high frequency $(1 \mathrm{~Hz}$ for $900 \mathrm{~s}$, at $10 \mathrm{~Hz}$ for $50 \mathrm{~s}$ at an interval of $50 \mathrm{~s}$ with 900 pulses in total) [21]. No stimulation was given to the neurons for control purpose. Incubation was allowed for $3 \mathrm{~h}$ prior to subsequent experiments. Rats treated with rTMS were subjected to rTMS treatment 1 time per day for 2 consecutive weeks at $24 \mathrm{~h}$ after surgery. Rats were stimulated using the standard figure-of-eight coil (MagPro Magnetic Stimulator, Farum, Denmark), fixed and oriented to cause synaptic activity in most cortical areas of the left hemisphere. During the stimulation, rats were un-anesthetized, wrapped, and gently fixed on a platform using Velcro straps. Their eyes were partly covered with a thick cloth. Next, the rats were allowed to acclimatize to the condition 1 week prior to the surgery in order to relieve the pressure associated with handling, fixing, and stimulation [22]. All rats were allowed free access to food and drinking water during the experiment.
Neurological evaluations were conducted by 2 blinded observers in the 1 st and 2 nd week of stimulation. The effect of rTMS in the first week was not obvious, but the effects in the second week were obvious. The grading process included the following: 1) neurological status in accordance with the methods reported by Bederson et al. [23] with 4 categories observed and noted ranging from 0 to 3 points in which $0=$ no neurological deficit observed, $1=$ contralateral forelimb flexion with wrist flexion and shoulder adduction, $2=$ reduced resistance to lateral push, and $3=$ circling movements towards the ipsilateral side; 2) wire grasping ability and forelimb strength scoring, ranging from 0 to 3 points that rats were suspended by its forelimbs on a wire stretched between two posts, $45 \mathrm{~cm}$ above a foam sheet, and the time was recorded until the rat fell down with the cutoff time set as $2 \mathrm{~min}$; and 3) learning-memory ability in which Ytype electric maze was implemented and the times of correct choice were recorded. Briefly, the elevated plus-maze apparatus (Inco) for rats was composed of a central platform connected to two open arms and two enclosed arms. The maze was elevated to a height of $50 \mathrm{~cm}$ above the floor. During training trials, the rats were placed at the end of an open arm with their faces turning away from the central platform of the maze. The time that the rats spent to move from open arm and cross the line marked in enclosed arm with all four paws was recorded as transfer latency (TL) time. If the rat did not enter the enclosed arm within $90 \mathrm{~s}$, it was gently pushed into the enclosed arm and the TL time was set as $90 \mathrm{~s}$. Then, the rats were allowed to stay in the maze for $10 \mathrm{~s}$. The TL measured on the plus maze on the 1st day was considered as the index of acquisition, whereas the TL measured after $24 \mathrm{~h}$ of acquisition trial was considered as the index of retrieval [24].

\section{Immunofluorescence}

The paraffin-embedded brain tissue slices were placed at $60{ }^{\circ} \mathrm{C}$ for $1 \mathrm{~h}$ until the paraffin thawed, followed by dewaxing and hydration using xylene and ethanol. After performing antigen retrieval by microwave oven irradiation for 9 min with high heat ( 3 times), the slices were allowed to cool down to room temperature. After being blocked with $5 \%$ goat serum at $37^{\circ} \mathrm{C}$ for $30 \mathrm{~min}$, the slices were stained with $5 \mathrm{mg} / \mathrm{mL}$ propidium iodide (PI) for 5 min, incubated with $0.5 \%$ Triton $\mathrm{X}-100$ at $37{ }^{\circ} \mathrm{C}$ for $30 \mathrm{~min}$, and probed with the primary rat antibody to hexaribonucleotide binding protein-3 (NeuN) (dilution ratio of 1:1000, ab104225, Abcam Inc., Cambridge, UK) at $4{ }^{\circ} \mathrm{C}$ overnight, followed by rewarming at $37^{\circ} \mathrm{C}$ for $1 \mathrm{~h}$. The sample was allowed to bind to the fluorescence 
secondary antibody (murine-green) (dilution ratio of 1:1000, ab150117, Abcam Inc., Cambridge, UK) for $2 \mathrm{~h}$, stained with $5 \mu \mathrm{g} / \mathrm{mL} 4^{\prime}$,6-diamidino-2-phenylindole (DAPI) for $5 \mathrm{~min}$ and stored at $4{ }^{\circ} \mathrm{C}$ in dark conditions. Observation was performed under a laser scanning confocal microscope (Olympus Corp., Tokyo, Japan). Subsequently, the ratio of NeuN/PI positive cells to NeuN positive cells was calculated and recorded.

\section{2,3,5-Triphenyl Tetrazolium Chloride Staining}

2,3,5-Triphenyl tetrazolium chloride (TTC) staining was applied to visualize the ischemic infarction. The fresh brain tissues were stored at $-20{ }^{\circ} \mathrm{C}$ for $10 \mathrm{~min}$, sectioned into 1-mm slices, and incubated with $1 \%$ TTC PBS (pH 7.4) at $37{ }^{\circ} \mathrm{C}$ for $20 \mathrm{~min}$ with gentle shaking. The slices were then fixed in $10 \%$ formaldehyde overnight, followed by photography using a digital camera. The living tissues were brick red-stained and cerebral infarction area exhibited grayish white-stained. The percentage of infarction area in total area was analyzed using ImagemasterVDS. The cerebral infarction area $(\%)=$ cerebral infarction area/total area $\times 100 \%$.

\section{RNA Isolation and Quantitation}

Primary neurons before and after repetitive magnetic stimulation (rMS) stimulation and rat tissues treated with

Table 1 Primer sequences for RT-qPCR

\begin{tabular}{ll}
\hline Gene & Primer sequences \\
\hline BDNF & F: 5'-AGACATGTTTGCGGCATCCAG-3' \\
& R: 5'-CCATAAGGACGGGGACTTGTAC-3' \\
BDNF exon I mRNA & F: 5'-CTCAAAGGGAAACGTGTCTCT-3' \\
& R: 5'-TCACGTGCTCAAAAGTGTCAG-3' \\
BDNF exon II mRNA & F: 5'-CTAGCCACCGGGGTGGTGTAA-3' \\
& R: 5'-TCACGTGCTCAAAAGTGTCAG-3' \\
BDNF exon IV mRNA & F: 5'-TGCGAGTATTACCTCCGCCAT-3' \\
& R: 5'-TCACGTGCTCAAAAGTGTCAG-3' \\
BDNF exon VI mRNA & F: 5'-TTGGGGCAGACGAGAAAGCGC-3' \\
& R: 5'-TCACGTGCTCAAAAGTGTCAG-3' \\
BDNF exon IX mRNA & F: 5'-GAGAAGAGTGATGACCATCCT-3' \\
& R: 5'-TCACGTGCTCAAAAGTGTCAG-3' \\
Tubulin, beta 4 mRNA & F: 5'-AGCAACATGAATGACCTGGTG-3' \\
& R: 5'-GCTTTCCCTAACCTGCTTGG-3' \\
\hline
\end{tabular}

RT-qPCR = reverse transcription quantitative polymerase chain reaction, $\mathrm{BDNF}=$ brain-derived neurotrophic factor, $\mathrm{mRNA}=$ messenger RNA, $\mathrm{F}$ $=$ forward, $\mathrm{R}=$ reverse
rTMS were collected and treated with $1 \mathrm{~mL}$ Trizol (Invitrogen, Carlsbad, CA, USA) in ice bath. Total RNA content was extracted using a RNA extraction kit (QIGEN, Duesseldorf, Germany). The concentration and purity of the obtained RNA were detected using an ultraviolet spectrophotometry (UV1901, Shanghai AuCy Instrument Co., Ltd., Shanghai, China) to ensure that the value of A260/A280 was between 1.8 and 2.0 and the concentration being $50 \mathrm{ng} / \mu \mathrm{L}$. Then, RNA was reverse transcribed into cDNA $(50 \mathrm{ng} / \mu \mathrm{L})$ according to the instructions of PrimeScriptTM RT reagent Kit (Takara, RR047A, Beijing Think-Far Technology Co., Ltd., Beijing, China). Primer sequences (Table 1) were designed by primer Premier 5.0 and synthesized by Beijing Tsingke Biological Technology Co., Ltd. (Beijing, China). The reaction was performed using the two-step method on an ABI 7900HT real-time quantitative PCR instrument (ABI 7900, Shanghai Pudi Biotechnology Co., Ltd., Shanghai, China) with $\beta$-actin serving as the internal reference. The expression of total mRNA and mRNA of BDNF exons was calculated using the $2^{-\Delta \Delta C t}$ method. Three replicates were set for each gene in each sample.

\section{Western Blot Analysis}

Total protein content was extracted from primary neurons before and after rMS stimulation and rat tissues using Radio Immunoprecipitation Assay lysis (R0010, Solarbio Life Sciences Co., Ltd., Beijing, China) containing phenylmethanesulfonyl fluoride, followed by incubation on ice for $30 \mathrm{~min}$. The protein (supernatant) was collected after centrifugation at $12000 \times \mathrm{g}$ for $10 \mathrm{~min}$ at $4{ }^{\circ} \mathrm{C}$ for quantification. The collected protein was separated with $10 \%$ SDS-polyacrylamide gel electrophoresis, and electroblotted onto a polyvinylidene fluoride membrane. Following blockade with 5\% skimmed milk powder at room temperature for $1 \mathrm{~h}$, the membrane was probed at $4{ }^{\circ} \mathrm{C}$ overnight with diluted primary rabbit polyclonal antibodies against BDNF (dilution ratio of 1:5000, ab108319, Abcam Inc., Cambridge, UK), RACK1 (dilution ratio of 1:5000, ab62735, Abcam Inc., Cambridge, UK), p-MeCP2 (dilution ratio of $1 \mathrm{mg} / \mathrm{mL}, \mathrm{IC} 17598$, Shanghai YuBo Biological Technology Co., Ltd., Shanghai, China), and $\beta$-actin (dilution ratio of 1:1000, ab8227, Abcam Inc., Cambridge, UK). After being rinsed by Tris-Buffered Saline and Tween 20 (TBST) for 3 times ( 5 min each time), the membrane was then probed with secondary horseradish peroxidase-labeled goat anti-rabbit Immunoglobulin $G$ antibody (dilution ratio of 1:1000, HA1003, Shanghai Yanhui Biotechnology Co., Ltd., 
Table 2 Neurobehavioral assessment in rats post-modeling in the presence of rTMS

\begin{tabular}{llllc}
\hline Group & $n$ & Neurological deficit score & Wire grasping ability score & The correct choice (n/min) \\
\hline Mock & 5 & $0.20 \pm 0.45$ & $2.80 \pm 0.45$ & $13.60 \pm 1.14$ \\
MCAO & 5 & $2.80 \pm 0.45$ & $0.20 \pm 0.45$ & $3.20 \pm 0.45$ \\
MCAO $+1 \mathrm{~Hz}$ & 5 & $1.20 \pm 0.84^{*}$ & $1.80 \pm 0.84^{*}$ & $7.40 \pm 0.89^{*}$ \\
$\mathrm{MCAO}+10 \mathrm{~Hz}$ & 5 & $0.60 \pm 0.55^{*}$ & $2.20 \pm 0.48^{*}$ & $12.20 \pm 1.30^{*}$ \\
\hline
\end{tabular}

${ }^{*} p<0.05 v s$ the MCAO group (rats with MCAO alone). MCAO = middle cerebral artery occlusion, rTMS $=$ repetitive transcranial magnetic stimulation
Shanghai, China) for $1 \mathrm{~h}$. Then, the enhanced chemiluminescence method was applied to development for $1 \mathrm{~min}$. The harvested protein was analyzed by Western blot analysis with $\beta$-actin serving as the internal reference [25].

\section{Chromatin Immunoprecipitation Assay}

The cortical tissues were cut into 2-mm slices, crosslinked by $1 \%$ formaldehyde, and stored at $-80{ }^{\circ} \mathrm{C}$ for further use. The nuclei were obtained by micro-probe, lysed, and treated with ultrasound until DNA fragment length was between 200 and $1000 \mathrm{bp}$. Protein G beads $(40 \mu \mathrm{L})$ were incubated with $3 \mu \mathrm{g}$ antibodies for at least $1 \mathrm{~h}$, followed by overnight incubation for immunoprecipitation. DNA-protein complex was extracted from beads and de-crosslinked at $65^{\circ} \mathrm{C}$ overnight. DNA was detached by RNase A and Proteinase K, extracted by phenol/ chloroform and precipitated with alcohol. Quantitative real-time PCR was performed using iQ SYBR Green Supermix (Bio-Rad Laboratories Inc., Hercules, CA, USA). The amount of DNA after immunoprecipitation was calculated by comparison to the amount of total input DNA. Each data was obtained from 4 times of independent amplification. Chromatin immunoprecipitation (ChIP) was conducted using RACK1 antibody (dilution ratio of 1:5000, ab62735, Abcam Inc., Cambridge, UK). The quantification of BDNF exons after precipitation was carried out by reverse transcription quantitative polymerase chain reaction (RT-qPCR).

\section{Statistical Analysis}

Statistical analyses were conducted using the SPSS 21.0 statistical software (IBM Corp. Armonk, NY, USA). If measurement data conformed to normal distribution and homogeneity of variance, measurement data were expressed as mean \pm standard deviation and analyzed by one-way analysis of variance among multiple groups, followed by Tukey's post hoc test. As for measurement data with skewed distribution, they were expressed as inter-quartile range and analyzed by Mann-Whitney $U$ test. A value of $p<0.05$ was considered to be statistically significant.

\section{Results}

\section{rMS Promotes BDNF Expression in Primary Cultured Neurons Treated with OGD}

Initially, primary cultured neurons obtained from the cortex, hippocampus, and striatum were subjected to OGD conditions to investigate the effects of rMS on BDNF expression. After OGD treatment, the BDNF expression was found to decrease significantly (Fig. 1A, B). Then, OGD-treated primary cultured neurons were subjected to rMS of low and high frequency. The primary cultured neurons for controls were also placed under rMS without stimulation. Subsequently, RT-qPCR and Western blot analysis were used to determine the BDNF expression pattern. As shown in Fig. 1A, B, compared with neurons following OGD treatment alone, $1 \mathrm{~Hz}$ rMS and $10 \mathrm{~Hz}$ rMS both upregulated the BDNF expression in OGDtreated neurons $(p<0.05)$, with especially significant upregulation in the cortex. The above findings suggested that rMS promoted the BDNF expression in OGDtreated primary cultured neurons.

\section{rMS Regulates the Transcription of BDNF exons in Primary Cultured Neurons}

Next, the expression patterns of BDNF exons were examined by means of RT-qPCR. Primary cultured neurons of cortex, hippocampus, and striatum were subjected to OGD conditions, followed by rMS of low and high frequency. As illustrated in Fig. 2, both the $1 \mathrm{~Hz}$ rMS and $10 \mathrm{~Hz}$ rMS induced different expression patterns of different BDNF exons in neurons of cortex, hippocampus, and striatum, with the most significant change of $B D N F$ exon $I V$ noted in the primary neurons isolated from 
cortex. These results suggested that rMS regulated the transcription of BDNF exons in different neurons.

\section{siRNA-Mediated Depletion of RACK1 Downregulates the Transcription of BDNF exons in OGD-Treated Primary Cultured Neurons Stimulated by rMS}

After rMS treatment, Bdnf IV expression was the highest and most obvious in the primary neurons isolated from cerebral cortex. Thus, the primary neurons isolated from cerebral cortex were selected for subsequent experiments. After discovering that rMS regulated the transcription of $B D N F$ exons in different neurons, we elucidated whether RACK1 mediates the regulatory role of rMS in the transcription of BDNF exons in OGD-treated neurons. We attained knockdown of RACK1 by employing the siRNA approach. Firstly, we confirmed that the interference efficacy of siRACK1 by RT-qPCR and Western blot analysis. As compared with siNC-transfected neurons, the addition of siRACK1 was found to significantly reduce the expression of RACK1 $(p<0.05$, Fig. 3A, B). Next, we explored whether siRACK1 affected the expression of BDNF exons (Fig. 3C). RT-qPCR demonstrated that expression of $B D N F$ exons (I, II, IV, and VI) was significantly decreased in neurons after OGD treatment $(p<0.01)$. Additionally, after OGD treatment, no differences in expression of BDNF exons were noted between siRACK1-transfected neurons and siNC-transfected neurons $(p>0.05)$. Then, the effect of rMS on BDNF expression after knockdown of RACK1 was examined (Fig. 3D, E). After OGD treatment followed by either $1 \mathrm{~Hz}$ rMS or $10 \mathrm{~Hz}$ rMS, the BDNF expression was observed to be obviously decreased in siRACK1-transfected neurons as compared to siNC-transfected neurons $(p<0.05)$. Further, we examined by what means RACK1 affected the BDNF expression and the expression of exons (I, II, IV, VI) of BDNF in rMS-stimulated OGD neurons (Fig. $3 \mathrm{~F}, \mathrm{G})$. After $1 \mathrm{~Hz}$ or $10 \mathrm{~Hz}$ rMS treatment, BDNF expression in the OGD-treated neurons was found to be downregulated by siRACK1 treatment, especially the $B D N F$ exon $I V(p<0.05)$. Knockdown of RACK 1 significant decreased the expression of exons (I, II, IV, VI) of BDNF in the OGD-treated neurons after $1 \mathrm{~Hz}$ or $10 \mathrm{~Hz}$ rMS, and over-expression of RACK 1 resulted in
Fig. 1 rMS upregulates the expression of BDNF in primary cultured neurons exposed to OGD. (A) mRNA expression patterns of BDNF in cortex, hippocampus, and stratum determined by RT-qPCR. (B) Relative expression patterns of BDNF protein in cortical, hippocampus, and stratum determined by Western blot analysis relative to $\beta$-actin. * $p<0.05 v$ s the OGD group (neurons treated with OGD alone). $* * p<0.01 v s$ the OGD group (neurons treated with OGD alone). The measurement data were expressed as mean \pm standard deviation. Comparison among multiple groups was analyzed by one-way analysis of variance. The experiment was repeated 3 times independently
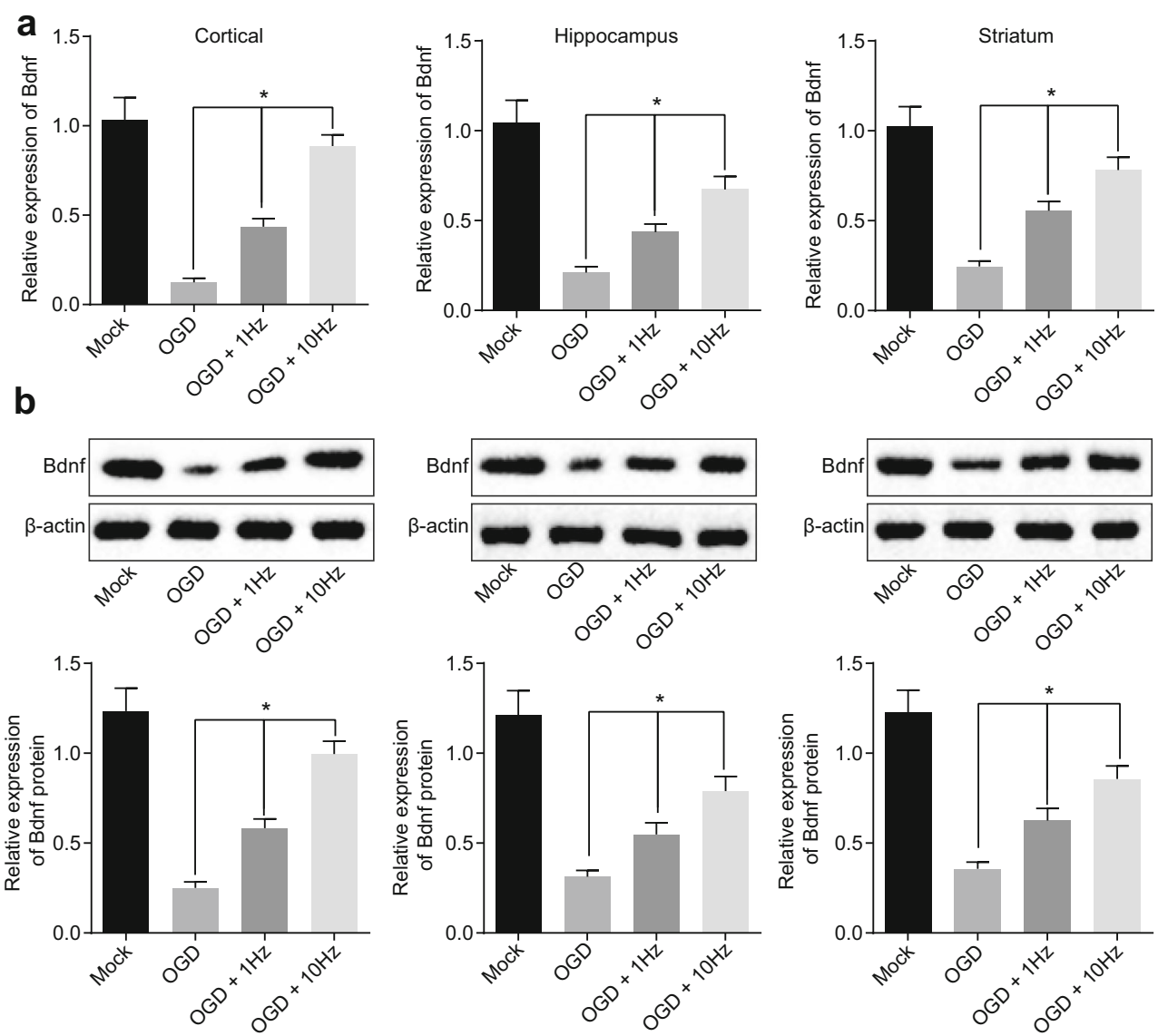

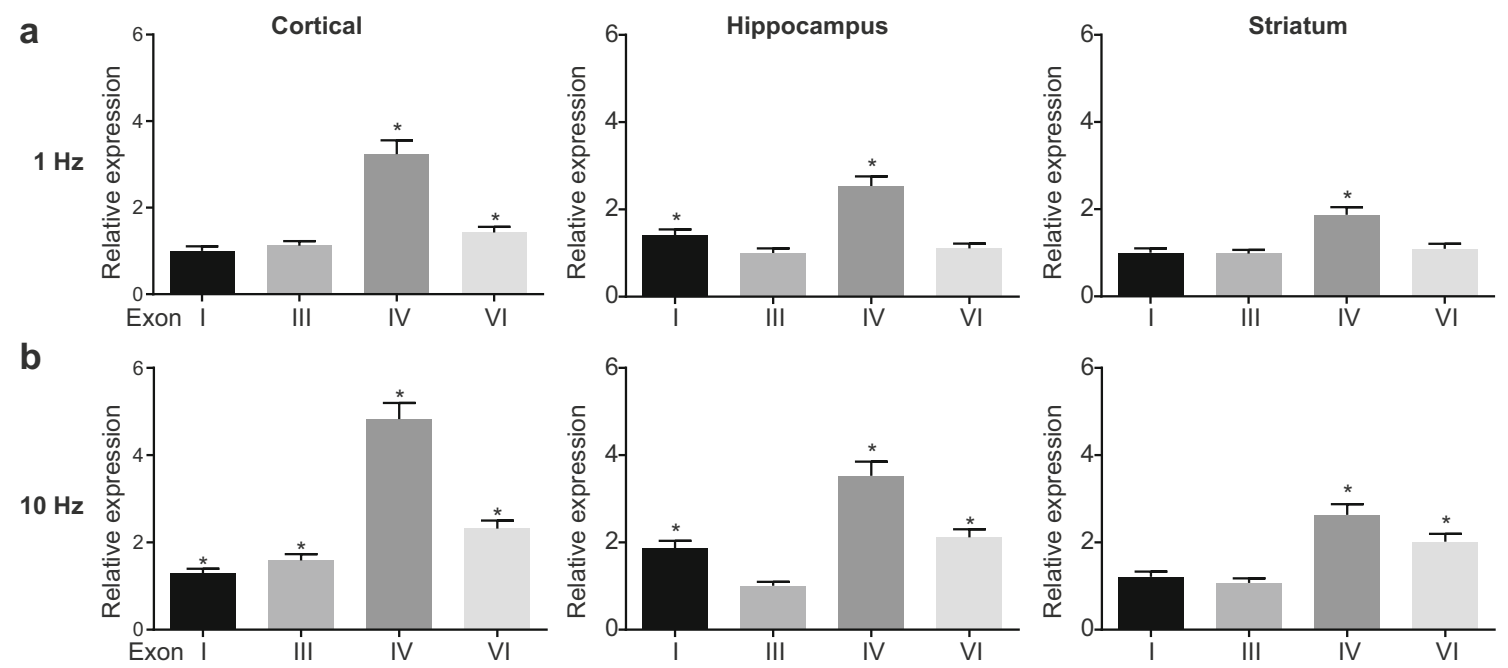

Fig. 2 rMS upregulates the transcription of different BDNF exons in primary cultured neurons. RT-qPCR was used to determine the relative expression patterns of different BDNF exons (I, II, IV, VI) in cortex, hippocampus, and stratum following $1 \mathrm{~Hz}$ or $10 \mathrm{~Hz}$ rMS. $* p<0.05 v \mathrm{~s}$ the OGD group (neurons treated with OGD alone). $* * p<0.01 v s$ the

OGD group (neurons treated with OGD alone). Measurement data were expressed as mean \pm standard deviation. Comparison among multiple groups was analyzed by one-way analysis of variance. The experiment was repeated 3 times independently

obviously increased expression, most strikingly in the $B D N F$ exon $I V(p<0.01)$. Therefore, BDNF exon $I V$ was selected for subsequent experimentation. These findings suggested that in the absence of rMS stimulation, downregulation of RACK1 exerted little effect on the transcription of BDNF exons in OGD-treated neurons, whereas RACK1 downregulation inhibited the transcription of $B D N F$ exons in OGD-treated primary cultured neurons in the presence of rMS stimulation.

\section{rMS Increases MeCP2 Phosphorylation to Promote the Interaction Between RACK1 and BDNF exon IV in Primary Cultured Neurons}

Then, we tested the involvement of MeCP2 in the interaction between RACK1 and BDNF exon IV. ChIP assay revealed that in comparison to OGD-treated neurons, $B D N F$ exon $I V$ was significantly enriched in OGDtreated neurons stimulated with $1 \mathrm{~Hz}$ or $10 \mathrm{~Hz}$ rMS $(p<0.05$, Fig. 4A). Western blot analysis demonstrated significantly higher levels of $\mathrm{MeCP} 2$ phosphorylation after $1 \mathrm{~Hz}$ or $10 \mathrm{~Hz}$ rMS compared to no rMS stimulation in OGD-treated neurons ( $p<0.05$, Fig. 4B). NR2A and NR2B are two subunits of NMDA receptor whose overexpression is associated with neurodegeneration [26, 27]. Then, in order to study how rMS regulates BDNF exon $I V$, NR2A specific inhibitor (NVP-AAM077) and NR2B specific inhibitor (Ro25-6981) were added prior to OGD and rMS treatments, followed by Western blot analysis to determine the levels of MeCP2 phosphorylation. We observed that NVP-AAM077 or Ro25-6981 led to a significant decrease in MeCP2 phosphorylation (Fig. 4C). Then, ChIP assay was performed again, which revealed that the addition of NVP-AAM007 or Ro25-6981 decreased the enrichment of $B D N F$ exon $I V$ in the presence of both OGD and $1 \mathrm{~Hz}$ or $10 \mathrm{~Hz}$ rMS (Fig. 4D). NVPAAM007 and Ro25-6981 inhibited the phosphorylation of $\mathrm{MeCP} 2$ induced by rMS stimulation, and the effect of $10 \mathrm{~Hz}$ rMS stimulation was more apparent. Furthermore, the effect of Ro25-6981 was more obvious than that of NVP-AAM077. Taken together, rMS increased MeCP2 phosphorylation, in turn improved the interaction between RACK1 and BDNF exon $I V$, eventually enhancing the expression of BDNF exon IV.

\section{rTMS of Different Frequencies Improves the Neurological Functions of Rats After MCAO}

Subsequently, in order to verify the improvement of neurologic functions caused by rTMS, we evaluated various neurobehavioral changes. As shown in Table $2,1 \mathrm{~Hz}$ or $10 \mathrm{~Hz}$ rTMS remarkably improved neurologic functions and abilities of movement balance, learning, and memory in MCAO rats $(p<0.05)$. Cell apoptosis analysis in the cortex of MCAO rats showed that $1 \mathrm{~Hz}$ or $10 \mathrm{~Hz}$ rTMS resulted in significantly lower apoptosis rates $(p<0.05$, Fig. 5A). TTC staining revealed that the percentage of cerebral infarction area was much smaller in the brain 

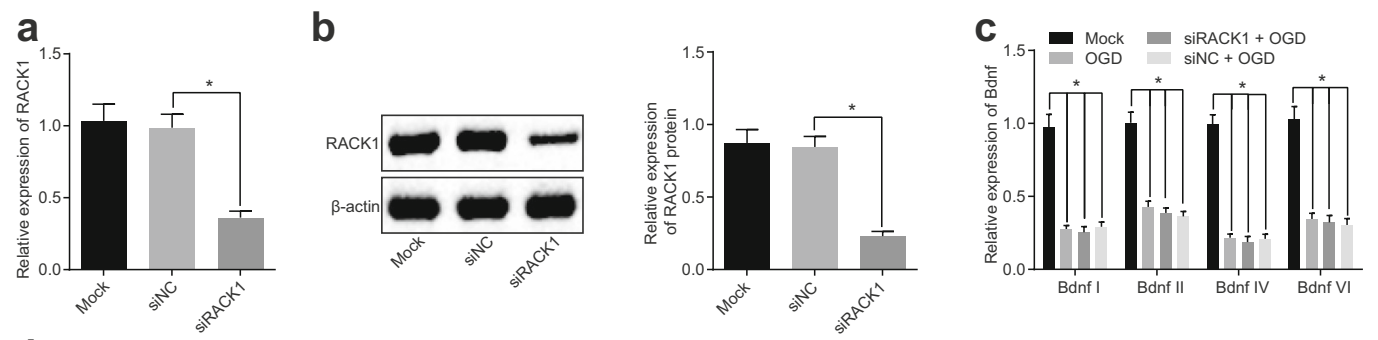

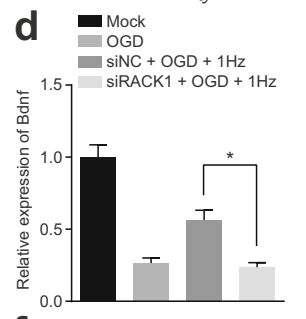

f $1.5-$ Mock $-\mathrm{siNC}+\mathrm{OGD}+1 \mathrm{~Hz}$
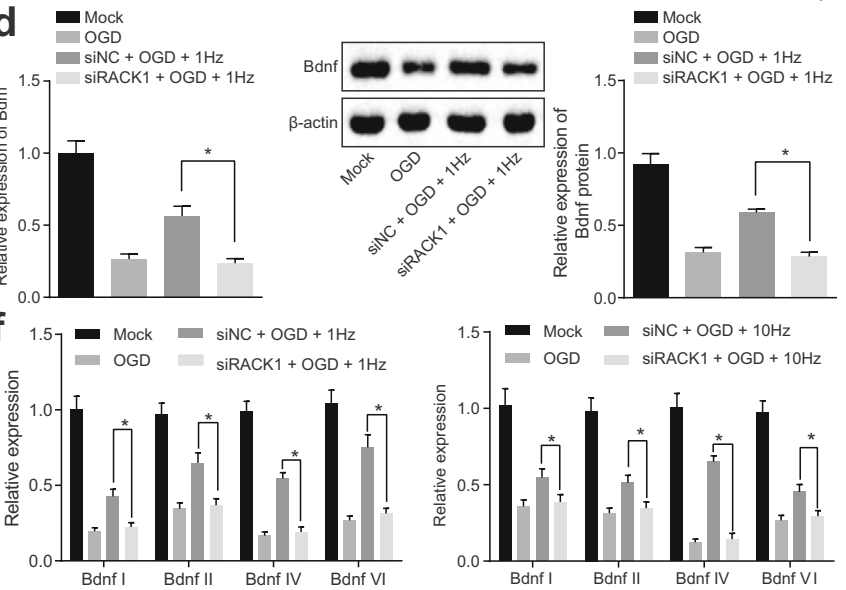

Fig. 3 Silencing of RACK1 inhibits the promoting effects of rMS on transcription of different $B D N F$ exons in OGD-treated primary cultured neurons. (A) The interference efficacy of siRACK1 determined by RTqPCR. (B) The interference efficacy of siRACK1 determined by Western blot analysis relative to $\beta$-actin. ${ }^{*} p<0.05 v$ s the siNC group (siNCtransfected neurons). (C) Relative expression patterns of different BDNF exons (I, II, IV, VI) in OGD-treated neurons in the presence of silenced RACK1 determined by RT-qPCR. $* p<0.05 v s$ the mock group (normal primary cultured neurons). (D) Relative expression patterns of BDNF following treatment of $1 \mathrm{~Hz}$ rMS, OGD, and siRACK1 determined by RT-qPCR and Western blot analysis, ${ }^{*} p<0.05 v s$ the siNC + OGD $+1 \mathrm{~Hz}$ group (neurons treated with all siNC, OGD, and $1 \mathrm{~Hz}$ rMS). (E) Relative expression patterns of BDNF following treatment of rMS of $10 \mathrm{~Hz}, \mathrm{OGD}$, and siRACK1 determined by RT-qPCR and Western blot

tissue from $1 \mathrm{~Hz}$ or $10 \mathrm{~Hz}$ rTMS-stimulated MCAO rats in comparison to MCAO rats in the absence of rTMS $(p<0.05$, Fig. 5B). In conclusion, low-frequency or high-frequency rTMS improved the neurologic functions in MCAO rat models.

\section{rTMS Promotes the Interaction Between RACK1 and BDNF exon IV by Enhancing MeCP2 Phosphorylation in a Rat Model of MCAO}

Finally, we speculated whether the action mechanisms of rTMS occurred in MCAO rat models as we observed in a cellular stroke model, that is, OGD cultured neurons. We selected the cortex in which rTMS directly act on. The extent of MeCP2 phosphorylation and BDNF exon $I V$ enriched by RACK1 was determined. We found higher levels of MeCP2 phosphorylation and BDNF exon $I V$

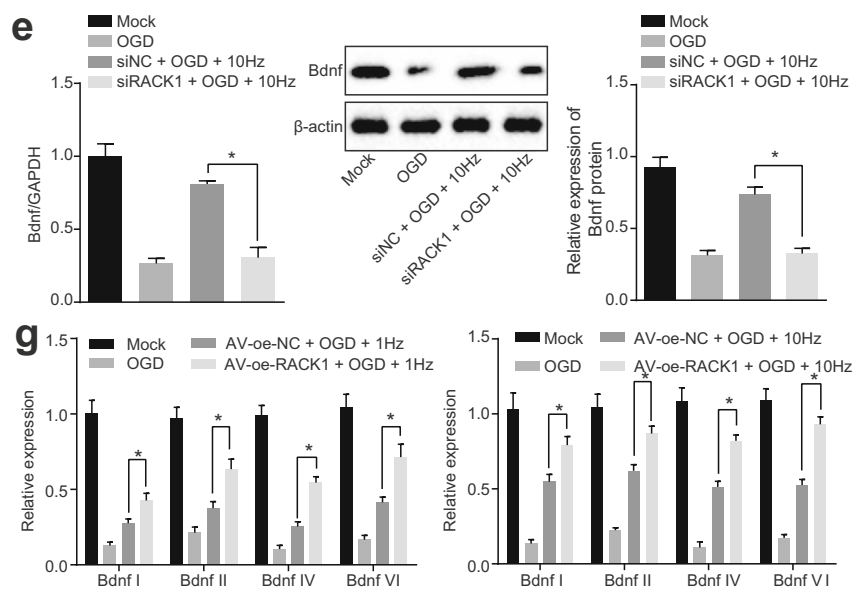

analysis. $* p<0.05 v s$ the siNC $+\mathrm{OGD}+10 \mathrm{~Hz}$ group (neurons treated with all siNC, OGD, and $10 \mathrm{~Hz}$ rMS). (F) Relative expression patterns of different $B D N F$ exons (I, II, IV, VI) following $1 \mathrm{~Hz}$ or $10 \mathrm{~Hz}$ rMS in the presence of silenced RACK1 determined by RT-qPCR. ** $p<0.05 v$ s the siNC $+\mathrm{OGD}+1 \mathrm{~Hz}$ or siNC $+\mathrm{OGD}+10 \mathrm{~Hz}$ group (neurons treated with all siNC, OGD, and $1 \mathrm{~Hz}$ or $10 \mathrm{~Hz}$ rMS). (G) Relative expression patterns of different $B D N F$ exons (I, II, IV, VI) following $1 \mathrm{~Hz}$ or $10 \mathrm{~Hz}$ rMS in the presence of over-expressed RACK1 determined by RT-qPCR. ${ }^{* *} p<0.01$ $v s$ the $\mathrm{AV}$-oe-NC + OGD $+1 \mathrm{~Hz}$ or $\mathrm{AV}$-oe-NC + OGD $+10 \mathrm{~Hz}$ group (neurons treated with all AV-oe-NC, OGD, and $1 \mathrm{~Hz}$ or $10 \mathrm{~Hz}$ rMS). Measurement data were expressed as mean \pm standard deviation. Comparison among multiple groups was analyzed by one-way analysis of variance. The experiment was repeated 3 times independently

enriched by RACK1 in MCAO rats stimulated by rTMS $(1 \mathrm{~Hz}$ or $10 \mathrm{~Hz})$ relative to MCAO rats without rTMS $(p<0.05$, Fig. 6A, B). Application of NR2A-specific inhibitor (NVP-AAM077) or NR2B-specific inhibitor (Ro25-6981) blocked the increase in MeCP2 phosphorylation levels and BDNF exon $I V$ enrichment caused by rTMS $(p<0.05$, Fig. $6 \mathrm{C}, \mathrm{D})$. Thus, rTMS promoted the interaction between RACK 1 and BDNF exon $I V$ by increasing MeCP2 phosphorylation in MCAO rat models.

\section{Discussion}

In recent years, mounting evidence has revealed rTMS as a promising therapeutic tool in neuromodulation, such as dysphagia after stroke, psychiatric disorders, and cerebral functional reorganization in ischemic stroke [28-30]. Considering 

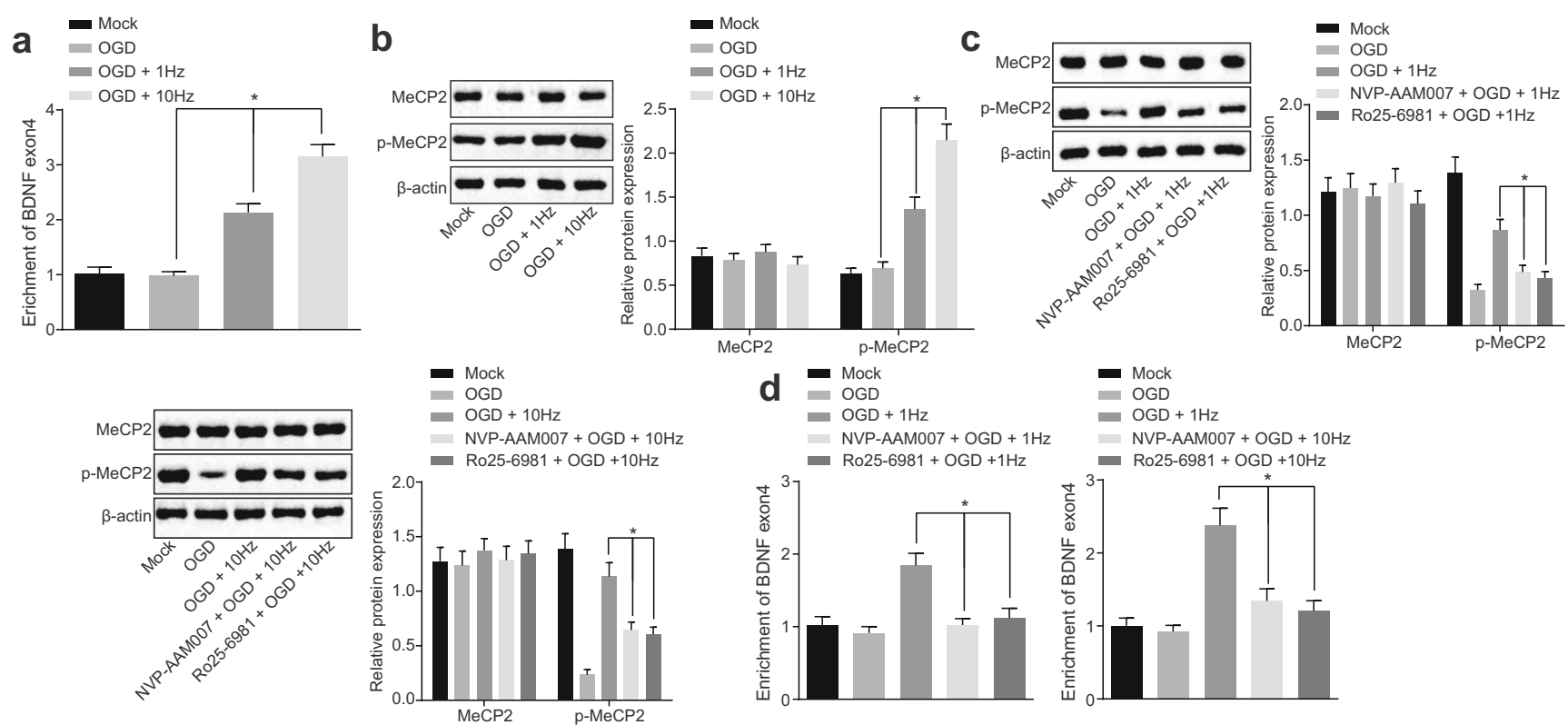

Fig. 4 rMS enhances the interaction between RACK 1 and BDNF exon IV by promoting the extent of MeCP2 phosphorylation. (A) Enrichment of BDNF exon IV by RACK1 detected by ChIP assay. (B) Extent of MeCP2 phosphorylation after rMS treatment detected by Western blot analysis relative to $\beta$-actin. $* p<0.05$ vs the OGD group (neurons treated with OGD alone). (C) Extent of MeCP2 phosphorylation in the presence of NR2A specific inhibitor (NVP-AAM077) or NR2B specific inhibitor (Ro25-6981) detected by Western blot analysis relative to $\beta$-actin. (D)

the limitation of in-depth investigation of the molecular mechanisms underlying rMS and rTMS, the current study examined the OGD-treated neurons and rat models of cerebral ischemia and confirmed the neuroprotective effect of rTMS on cerebral ischemia. The mechanism was speculated to involve the interaction between RACK1 and BDNF exon $I V$ by $\mathrm{MeCP} 2$ phosphorylation.

Initially, the current study demonstrated that rMS and rTMS upregulated the expression of BDNF in primary cultured neurons with OGD and MCAO rats in which the transcription of BDNF exon $I V$ was promoted to interact with RACK1 by MeCP2 phosphorylation. BDNF exon $I V$ has been regarded as a leading cause of BDNF expression depending on neuronal activity and RACK1 can regulate the exon-specific expression of BDNF expression as a transcription regulator [18]. In addition, RACK1 has been recognized to regulate chromatin remodeling, leading to the exon-specific expression of BDNF, the mechanism of which might influence the mediation of neuronal functions [18]. A previous study has pointed out that BDNF upregulation can be induced by MeCP2 phosphorylation [31]. Another study speculated that the methylation of BDNF exon IV CpG-87 may cause the specific $\mathrm{MeCP} 2$ binding [32]. In consistency with our results, significantly higher BDNF mRNA expression has been

Enrichment of BDNF exon $I V$ by RACK1 in the presence of NR2A specific inhibitor (NVP-AAM077) or NR2B specific inhibitor (Ro256981) detected by ChIP assay. * $p<0.05 v s$ the OGD $+1 \mathrm{~Hz}$ or OGD + $10 \mathrm{~Hz}$ group (neurons treated with both OGD and $1 \mathrm{~Hz}$ or $10 \mathrm{~Hz}$ rMS). Measurement data were expressed as mean \pm standard deviation. Comparison among multiple groups was analyzed by one-way analysis of variance. The experiment was repeated 3 times independently

found in the cortex after rTMS than contralateral one [33]. Similarly, the mechanism of BDNF upregulation has been previously elucidated to be related to rTMS in the hippocampus [34], which was additionally confirmed in the current study.

Importantly, the MCAO-induced cerebral ischemia model in rats showed that the neuropsychological assessment results of rats treated with $1 \mathrm{~Hz}$ or $10 \mathrm{~Hz}$ rTMS were more satisfactory than those without rTMS treatment. Furthermore, our findings indicated that rTMS was effective in the treatment of cerebral ischemia as evidenced by lower neurological deficit scores, inhibited neuronal apoptosis, and smaller cerebral infarction area. Following ischemic insult, if neurons die, it is troublesome to reconstruct the damaged neural circuits, which is also the key to effective treatment [35]. Evidences have highlighted that the repeated magnetic pulses caused by rTMS are capable of modifying the neural activity and behavior and rTMS possesses the ability to reconstruct functions of the injured neural network [36, 37]. Largely in agreement with the observations of our study, a MCAOinduced rat model of transient cerebral ischemia following rTMS was observed with higher neurological scores and reduced infarct volumes of cortex and striatum [38]. Because the MCAO model of stroke is known to result 

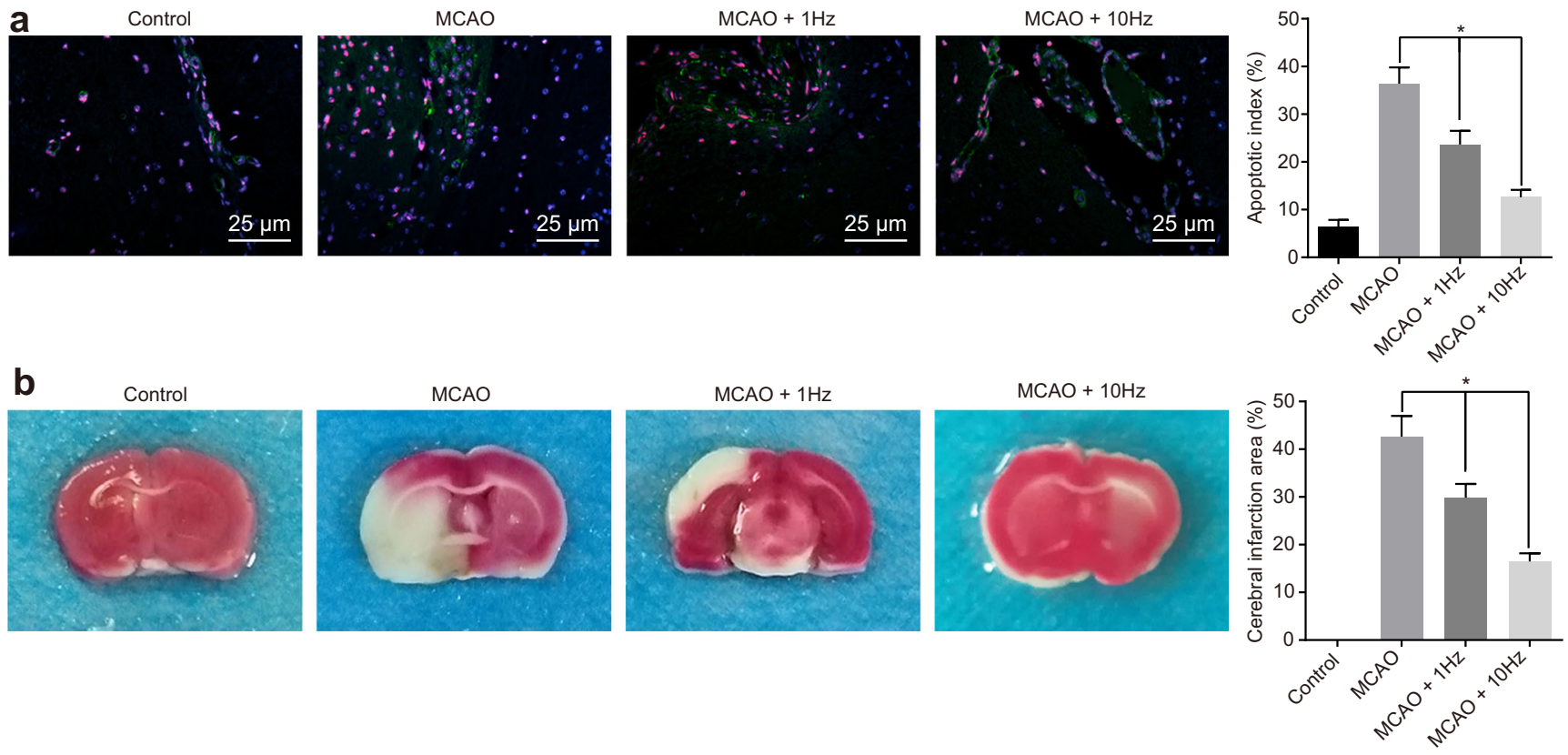

Fig. 5 rTMS of different frequencies leads to improved neurologic function in MCAO rats. (A) Neuron apoptosis in the cortex tissue identified by immunofluorescence $(\times 400)$. (B) Cerebral infarction area identified by TTC staining. $* p<0.05 v s$ the MCAO group (rats that

in heterogeneous infarcts of various sizes, therefore comparing infarct sizes post intervention in order to demonstrate neuroprotective effects is controversial. The large underwent MCAO). Measurement data were expressed as mean \pm standard deviation. Comparison among multiple groups was analyzed by oneway analysis of variance. $n=5$

differences in infarct size between treatment groups could even suggest a problem with randomization prior to treatment. Because neurological function largely depends on a

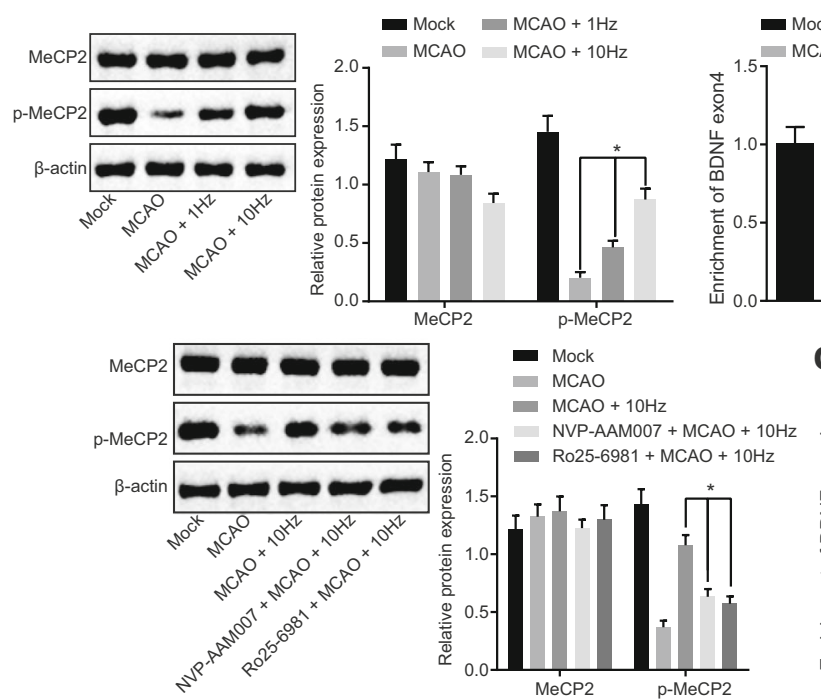

Fig. 6 rTMS promotes the interaction between RACK1 and BDNF exon $I V$ by increasing the extent of $\mathrm{MeCP} 2$ phosphorylation in MCAO rats. (A) Extent of MeCP2 phosphorylation detected by Western blot analysis relative to $\beta$-actin. (B) Enrichment of BDNF exon $I V$ by RACK1 detected by ChIP assay. $* p<0.05 v s$ the MCAO group (rats that underwent MCAO alone). (C) Extent of MeCP2 phosphorylation in the presence of NR2A specific inhibitor (NVP-AAM077) or NR2B specific inhibitor (Ro25-6981)

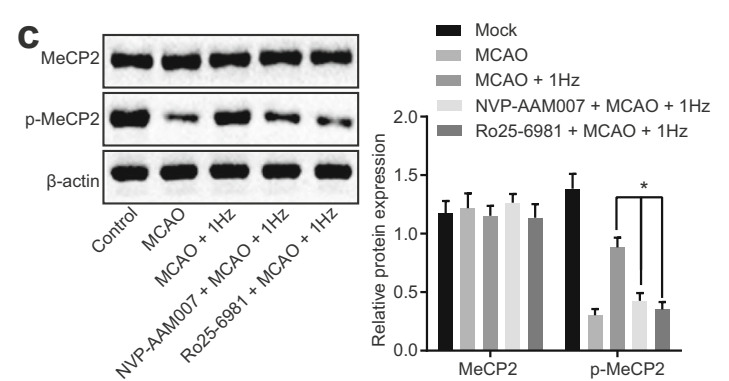

\section{d Mock \\ MC \\ MCAO $+1 \mathrm{~Hz}$}

I.5 NVP-AAMO07+ MCAO + $1 \mathrm{~Hz}$

व. $\mathrm{Ro} 25-6981+\mathrm{MCAO}+1 \mathrm{~Hz}$

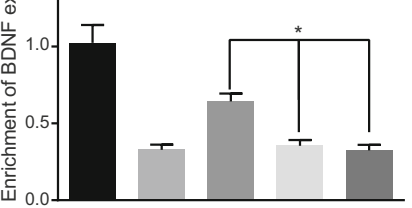

Mock

- MCAO

- $\mathrm{MCAO}+10 \mathrm{~Hz}$

$1.5 \quad$ NVP-AAMOO7 + MCAO $+10 \mathrm{~Hz}$

$\left.\stackrel{\mathrm{C}}{0}^{1.5}\right]=\mathrm{Ro} 25-6981+\mathrm{MCAO}+10 \mathrm{~Hz}$

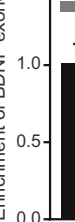

T
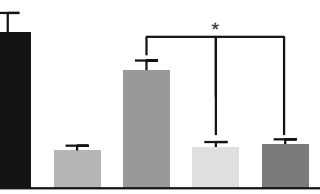

detected by Western blot analysis relative to $\beta$-actin. (D) Enrichment of $B D N F$ exon $I V$ by RACK1 in the presence of NR2A specific inhibitor (NVP-AAM077) or NR2B specific inhibitor (Ro25-6981) detected by ChIP assay. $* p<0.05 v s$ the MCAO $+1 \mathrm{~Hz}$ or MCAO $+10 \mathrm{~Hz}$ group (rats that underwent MCAO and $1 \mathrm{~Hz}$ or $10 \mathrm{~Hz}$ rTMS). Measurement data were expressed as mean \pm standard deviation. Comparison among multiple groups was analyzed by one-way analysis of variance. $n=5$ 
Fig. 7 Mechanistic diagram depicting the effect of rTMS on cerebral ischemia via BDNF. In cerebral ischemia, rTMS increases the extent of $\mathrm{MeCP} 2$ phosphorylation, further promoting the interaction between RACK1 and BDNF exon $I V$, whereby neurologic deficits are improved

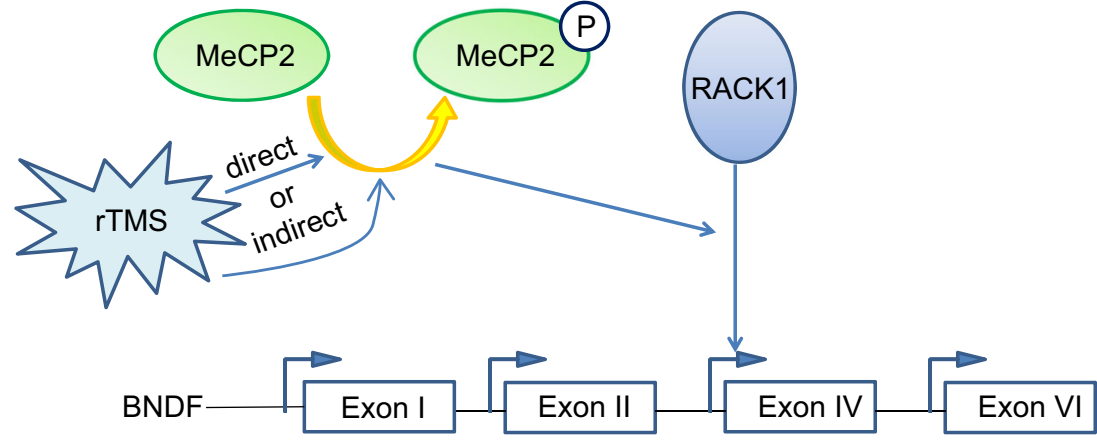

lesion size, these thoughts apply to the behavioral data as well. However, owing to limited time and cost, our study fails to further demonstrate these issues with a comment on technicality and study design, which will be the focus of our future study. Likewise, the anti-apoptotic property of rTMS has been illustrated in rat models of subacute cerebral ischemia [39], supporting the presence of a regulatory mechanism of rTMS in cerebral ischemia. Additionally, another study indicated that the motor function and disabilities caused by ischemic stroke can be improved sustainably by $1 \mathrm{~Hz}$ or $5 \mathrm{~Hz}$ rTMS [40]. Notably, low-frequency rTMS $(1 \mathrm{~Hz})$ has been considered to be efficient in patients suffering from aphasia after the occurrence of stroke [41]. The frequency-specific influences on the nodes of the motor network induced by rTMS have been identified to exert its optimal effects at a frequency of $5 \mathrm{~Hz}$ [42]. However, in an ischemic rat model, better effects were detected considering neurogenesis enhancement with frequency of rTMS being $20 \mathrm{~Hz}$ via the BDNF/TrkB signaling pathway [6]. The circumstantial evidence in our study suggested that the application of a stimulation of $10 \mathrm{~Hz}$ was better than $1 \mathrm{~Hz}$, indicating the need of further research to determine the optimum frequency. Moreover, a 6-year follow-up research of patients with depression that underwent rTMS shows the great clinical significance of rTMS considering its advantages of safety and efficacy, highlighting the potential role of rTMS as a useful treatment alternative [43].

Taken together, the findings of the current study strongly support the notion that rTMS exerts neuroprotective effects in cerebral ischemia and is capable of improving neurological deficits by promoting the interaction between RACK1 and BDNF exon $I V$ through the increase of the extent of MeCP2 phosphorylation (Fig. 7). Ultimately, our study sheds new light on a novel therapeutic target for future treatment of cerebral ischemia from on-bench evidence to clinical situations. However, rTMS protocols deserve more rigorous researches to optimize technical realization and to provide proper care of patients as well as to increase success rate [44], thereby taking the findings of the study to the next level theoretically or practically.

Acknowledgments We acknowledge and appreciate our colleagues for their valuable efforts and comments on this paper.

Authors' Contributions HZL and JQS designed the study. CLZ collated the data and designed and developed the database. RLL and JPC carried out data analyses and produced the initial draft of the manuscript. HZL, JQS, and XJZ contributed to drafting the manuscript. All authors contributed to the revision and approved the final manuscript.

Funding This work was funded by National Natural Science Foundation of China (No.81671284 to XZ \& No.81471338).

\section{Compliance with Ethical Standards}

Animal use and experimental procedures were carried out with the approval of the Experimental Animal Ethics Committee of Integrated Hospital of Traditional Chinese Medicine, Southern Medical University and the Affiliated Changzhou No. 2 People's Hospital of Nanjing Medical University. All experimental animals operating procedures strictly conformed to the recommendations in the Guide for the Care and Use of Laboratory Animals. Best efforts were made to minimize the suffering of the included animals

Conflict of Interest The authors declare that they have no conflict of interest.

\section{References}

1. Wang P, Xu TY, Wei K, Guan YF, Wang X, Xu H, et al. ARRB1/ beta-arrestin-1 mediates neuroprotection through coordination of BECN1-dependent autophagy in cerebral ischemia. Autophagy. 2014;10(9):1535-48.

2. Goncalves LV, Herlinger AL, Ferreira TAA, Coitinho JB, Pires RGW, Martins-Silva C. Environmental enrichment cognitive neuroprotection in an experimental model of cerebral ischemia: biochemical and molecular aspects. Behav Brain Res 2018;348(17183.

3. Martin DM, McClintock SM, Forster J, Loo CK. Does Therapeutic Repetitive Transcranial Magnetic Stimulation Cause Cognitive Enhancing Effects in Patients with Neuropsychiatric Conditions? 
A Systematic Review and Meta-Analysis of Randomised Controlled Trials. Neuropsychol Rev 2016;26(3):295-309.

4. Motta C, Di Lorenzo F, Ponzo V, Pellicciari MC, Bonni S, Picazio $\mathrm{S}$, et al. Transcranial magnetic stimulation predicts cognitive decline in patients with Alzheimer's disease. J Neurol Neurosurg Psychiatry 2018;89(12):1237-42.

5. Guo F, Han X, Zhang J, Zhao X, Lou J, Chen H, et al. Repetitive transcranial magnetic stimulation promotes neural stem cell proliferation via the regulation of MiR-25 in a rat model of focal cerebral ischemia. PLoS One 2014;9(10):e109267.

6. Luo J, Zheng H, Zhang L, Zhang Q, Li L, Pei Z, et al. HighFrequency Repetitive Transcranial Magnetic Stimulation (rTMS) Improves Functional Recovery by Enhancing Neurogenesis and Activating BDNF/TrkB Signaling in Ischemic Rats. Int J Mol Sci 2017;18(2):

7. Berretta A, Tzeng YC, Clarkson AN. Post-stroke recovery: the role of activity-dependent release of brain-derived neurotrophic factor. Expert Rev Neurother 2014;14(11):1335-44.

8. Guo F, Lou J, Han X, Deng Y, Huang X. Repetitive Transcranial Magnetic Stimulation Ameliorates Cognitive Impairment by Enhancing Neurogenesis and Suppressing Apoptosis in the Hippocampus in Rats with Ischemic Stroke. Front Physiol 2017;8(559.

9. Ceci M, Welshhans K, Ciotti MT, Brandi R, Parisi C, Paoletti F, et al. RACK1 is a ribosome scaffold protein for beta-actin mRNA/ ZBP1 complex. PLoS One 2012;7(4):e35034.

10. Cheng M, Xue H, Cao W, Li W, Chen H, Liu B, et al. Receptor for Activated C Kinase 1 (RACK1) Promotes Dishevelled Protein Degradation via Autophagy and Antagonizes Wnt Signaling. J Biol Chem 2016;291(24):12871-9.

11. Kershner L, Welshhans K. RACK1 regulates neural development. Neural Regen Res 2017;12(7):1036-9.

12. Gu L, Huang B, Shen W, Gao L, Ding Z, Wu H, et al. Early activation of nSMase2/ceramide pathway in astrocytes is involved in ischemia-associated neuronal damage via inflammation in rat hippocampi. J Neuroinflammation 2013;10(109.

13. Wang HY, Crupi D, Liu J, Stucky A, Cruciata G, Di Rocco A, et al. Repetitive transcranial magnetic stimulation enhances BDNF-TrkB signaling in both brain and lymphocyte. J Neurosci 2011;31(30): 11044-54.

14. Barneda-Zahonero B, Servitja JM, Badiola N, et al. Nurrl protein is required for N-methyl-D-aspartic acid (NMDA) receptor-mediated neuronal survival. J Biol Chem 2012;287(14): 11351-62.

15. Ebert DH, Gabel HW, Robinson ND, Kastan NR, Hu LS, Cohen S, et al. Activity-dependent phosphorylation of MeCP2 threonine 308 regulates interaction with NCoR. Nature. 2013;499(7458):341-5.

16. van der Vaart M, Svoboda O, Weijts BG, Espin-Palazon R, Sapp V, Pietri T, et al. Mecp2 regulates tnfa during zebrafish embryonic development and acute inflammation. Dis Model Mech 2017;10(12):1439-51.

17. Ausio J. MeCP2 and the enigmatic organization of brain chromatin. Implications for depression and cocaine addiction. Clin Epigenetics 2016;8(58.

18. He DY, Neasta J, Ron D. Epigenetic regulation of BDNF expression via the scaffolding protein RACK1. J Biol Chem 2010;285(25):19043-50.

19. Yan H, Yuan J, Gao L, Rao J, Hu J. Long noncoding RNA MEG3 activation of p53 mediates ischemic neuronal death in stroke. Neuroscience. 2016;337(191-9.

20. Jiang Y, Yang S, Tao J, Lin Z, Ye X, You Y, et al. Opposing needling promotes behavior recovery and exerts neuroprotection via the cAMP/PKA/CREB signal transduction pathway in transient MCAO rats. Mol Med Rep 2016;13(3):2060-70.
21. Funke K, Benali A. Modulation of cortical inhibition by rTMS findings obtained from animal models. J Physiol 2011;589(Pt 18): 4423-35.

22. Ljubisavljevic MR, Javid A, Oommen J, et al. The Effects of Different Repetitive Transcranial Magnetic Stimulation (rTMS) Protocols on Cortical Gene Expression in a Rat Model of Cerebral Ischemic-Reperfusion Injury. PLoS One 2015;10(10): e0139892.

23. Bederson JB, Pitts LH, Tsuji M, Nishimura MC, Davis RL, Bartkowski H. Rat middle cerebral artery occlusion: evaluation of the model and development of a neurologic examination. Stroke. 1986;17(3): 472-6.

24. Rakhunde PB, Saher S, Ali SA. Neuroprotective effect of Feronia limonia on ischemia reperfusion induced brain injury in rats. Indian J Pharm 2014;46(6): 617-21.

25. Shi Y, Jiang X, Zhang L, et al. Endothelium-targeted overexpression of heat shock protein 27 ameliorates blood-brain barrier disruption after ischemic brain injury. Proc Natl Acad Sci U S A 2017;114(7): E1243-E52.

26. Toyoda H, Zhao MG, Zhuo M. Roles of NMDA receptor NR2A and NR2B subtypes for long-term depression in the anterior cingulate cortex. Eur J Neurosci 2005;22(2):485-94.

27. Zhou X, Hollern D, Liao J, Andrechek E, Wang H. NMDA receptor-mediated excitotoxicity depends on the coactivation of synaptic and extrasynaptic receptors. Cell Death Dis 2013;4(e560.

28. Liao X, Xing G, Guo Z, Jin Y, Tang Q, He B, et al. Repetitive transcranial magnetic stimulation as an alternative therapy for dysphagia after stroke: a systematic review and meta-analysis. Clin Rehabil 2017;31(3):289-98.

29. Guo Q, Li C, Wang J. Updated Review on the Clinical Use of Repetitive Transcranial Magnetic Stimulation in Psychiatric Disorders. Neurosci Bull 2017;33(6):747-56.

30. Li J, Zhang XW, Zuo ZT, Lu J, Meng CL, Fang HY, et al. Cerebral Functional Reorganization in Ischemic Stroke after Repetitive Transcranial Magnetic Stimulation: An fMRI Study. CNS Neurosci Ther 2016;22(12):952-60.

31. Tadic A, Muller-Engling L, Schlicht KF, Kotsiari A, Dreimuller N, Kleimann A, et al. Methylation of the promoter of brain-derived neurotrophic factor exon IV and antidepressant response in major depression. Mol Psychiatry 2014;19(3):281-3.

32. Lieb K, Dreimuller N, Wagner S, Schlicht K, Falter T, Neyazi A, et al. BDNF Plasma Levels and BDNF Exon IV Promoter Methylation as Predictors for Antidepressant Treatment Response. Front Psychiatry 2018;9(511.

33. Beom J, Lee JC, Paeng JC, Han TR, Bang MS, Oh BM. Repetitive Transcranial Magnetic Stimulation to the Unilateral Hemisphere of Rat Brain. J Vis Exp 2016;116): e54217.

34. Zhang N, Xing M, Wang Y, Tao H, Cheng Y. Repetitive transcranial magnetic stimulation enhances spatial learning and synaptic plasticity via the VEGF and BDNF-NMDAR pathways in a rat model of vascular dementia. Neuroscience. 2015;311(284-91.

35. Zhu L, Wang L, Ju F, Khan A, Cheng X, Zhang S. Reversible recovery of neuronal structures depends on the degree of neuronal damage after global cerebral ischemia in mice. Exp Neurol 2017;289(1-8.

36. Chou YH, Hickey PT, Sundman M, Song AW, Chen NK. Effects of repetitive transcranial magnetic stimulation on motor symptoms in Parkinson disease: a systematic review and meta-analysis. JAMA Neurol 2015;72(4):432-40.

37. Feng HL, Yan L, Cui LY. Effects of repetitive transcranial magnetic stimulation on adenosine triphosphate content and microtubule associated protein-2 expression after cerebral ischemia-reperfusion injury in rat brain. Chin Med J 2008;121(14):1307-12. 
38. Gao F, Wang S, Guo Y, et al. Protective effects of repetitive transcranial magnetic stimulation in a rat model of transient cerebral ischaemia: a microPET study. Eur J Nucl Med Mol Imaging 2010;37(5): 954-61.

39. Yoon KJ, Lee YT, Han TR. Mechanism of functional recovery after repetitive transcranial magnetic stimulation (rTMS) in the subacute cerebral ischemic rat model: neural plasticity or anti-apoptosis? Exp Brain Res 2011;214(4): 549-56.

40. Emara TH, Moustafa RR, Elnahas NM, Elganzoury AM, Abdo TA, Mohamed SA, et al. Repetitive transcranial magnetic stimulation at $1 \mathrm{~Hz}$ and $5 \mathrm{~Hz}$ produces sustained improvement in motor function and disability after ischaemic stroke. Eur J Neurol 2010;17(9): 1203-9.

41. Li Y, Qu Y, Yuan M, Du T. Low-frequency repetitive transcranial magnetic stimulation for patients with aphasia after stoke: A metaanalysis. J Rehabil Med 2015;47(8):675-81.
42. Salinas FS, Franklin C, Narayana S, Szabo CA, Fox PT. Repetitive Transcranial Magnetic Stimulation Educes Frequency-Specific Causal Relationships in the Motor Network. Brain Stimul 2016;9(3):406-14.

43. Galletly CA, Clarke P, Carnell BL, Gill S. A clinical repetitive transcranial magnetic stimulation service in Australia: 6 years on. Aust N Z J Psychiatry 2015;49(11):1040-7.

44. Lefaucheur JP, Andre-Obadia N, Antal A, Ayache SS, Baeken C, Benninger DH, et al. Evidence-based guidelines on the therapeutic use of repetitive transcranial magnetic stimulation (rTMS). Clin Neurophysiol 2014;125(11):2150-206.

Publisher's Note Springer Nature remains neutral with regard to jurisdictional claims in published maps and institutional affiliations. 